\section{Spine fix for ALS}

\section{By Michael J. Haas, Senior Writer}

Researchers have shown in Nature Neuroscience that targeted transplantation of astrocyte precursor cells may extend the lifespan of patients with amyotrophic lateral sclerosis by slowing the loss of respiratory motor neuron function. ${ }^{1} \mathbf{Q}$ Therapeutics Inc. and some of the researchers are collaborating to develop the findings for ALS and other indications involving spinal cord degeneration.

ALS causes loss of sensation, loss of motor control and paralysis. Patients usually die three to five years after diagnosis, when degeneration of motor neurons leads to respiratory failure. Rilutek riluzole, a glutamate antagonist from sanofi-aventis Group, is the only approved ALS drug.

Studies over the last seven years have suggested that diseased nonneuronal cells such as astrocytes contribute to neuronal dysfunction and death in both forms of the disease. Familial ALS occurs in about 20\% of patients, and about one-fourth of such cases are linked to mutations in the gene coding for superoxide dismutase 1 (SOD1). Nonfamilial, sporadic ALS is the most common form.

In February, researchers at the University of California, San Diego reported that loss of motor neuron function was slower in mice whose astrocytes did not express mutant SOD1 than it was in control mice expressing mutant SOD1, confirming that astrocytes do play a role in the progression of ALS. ${ }^{2}$

The problem, however, is that ALS affects the entire spinal column, and as a result cell-based therapies have been deemed unfeasible, according to Jeffrey Rothstein, coauthor of the Nature Neuroscience paper. Rothstein is a professor of neurology and neuroscience and co-director of the Brain Science Institute at Johns Hopkins University.

"Instead of trying to treat the entire spinal column, we targeted the part of the spinal column that involves the respiratory motor neurons to address the main cause of mortality in ALS," he told SciBX.

The group was led by Nicholas Maragakis, associate professor of neurology at Johns Hopkins, and also included Mahendra Rao, who is cofounder and chief scientific consultant at Q Therapeutics and VP of stem cell research at Invitrogen Corp. In 1998, Rao and other researchers at the University of Utah School of Medicine developed the rodent astrocyte precursor cells. ${ }^{3}$

For their study, the Maragakis team used rodent cells called glialrestricted precursor cells, which can differentiate into only two types of cells: astrocytes, which support the functions of nearby neurons, or oligodendrocytes, which regulate neuron myelination.

The team from Johns Hopkins and Invitrogen reported that astrocyte precursor cells injected at multiple sites on the spines of rats with ALS differentiated into astrocytes and conferred a neuroprotective effect on nearby respiratory motor neurons. Treated rats survived for 173 days compared with 156 days for controls-a lifespan extension of 17 days. ${ }^{1}$

Immunohistological analysis showed that about one-third of the transplanted cells survived and differentiated into normal astrocytes that replaced the functions of diseased astrocytes near the site of injection.

The team also found a slower onset in the loss of forelimb motor control in the treated rats because the motor neurons governing respiration and forelimb control are close together in the rat spinal column, according to Maragakis. That finding suggested the transplant therapy "could target other areas of [the] spine in the same way-for instance, the lower spinal column in humans, where motor function of legs is involved," he told SciBX.

Additional murine studies showed that the transplants conferred a neuroprotective effect by maintaining normal levels of the glutamate transporter solute carrier family 1 (glial high affinity glutamate transporter), member 2 (Slc1a2; Eaat2; GLT1), which is the rodent homolog of the human protein SLC1A2, which is lost during ALS disease progression.

"Since 1995 this protein has been indicative of changes to astrocytes in sporadic, nonfamilial ALS-the most common form of the disease," said Rothstein.

Maragakis said that astrocyte precursor therapy should be effective in both familial ALS and sporadic, nonfamilial ALS. But, he noted, "there are no animal models of sporadic ALS to test this hypothesis."

\section{Restoration hardware}

In the same week that the Nature Neuroscience paper was published online, Q Therapeutics announced a collaboration with Johns Hopkins to treat ALS in humans with the company's Q-cells, which are human analogs of the precursor cells used by the Maragakis team. The company expects to submit an IND in 2009.

Deborah Eppstein, president and CEO at Q Therapeutics, said the strategy described in the paper was more practical than trying to repair or replace defective neurons, which is the focus of most research in neurodegenerative diseases.

In addition to bypassing the challenges of regenerating neurons, Eppstein said astrocyte transplants may provide benefits to neurons through multiple mechanisms. "There are so many things that are going wrong in a disease like ALS that it's hard to figure out exactly where to intervene with a targeted drug treatment, which is why we work with cells rather than drug molecules," she said.

"By putting back whole cells," Rothstein added, "not only do you treat the disease but you can also figure out which function or functions are restored by the cells." That could eventually lead to therapeutics that prevent or restore that loss of function, he said.

Eppstein said the precursor cells also could be used to screen for such therapeutics.

Richard Garr, president and CEO of NeuralStem Inc., said multiple injections of cells at different sites in the spinal column is likely the best approach for addressing loss of motor function in ALS. However, he 
does not think astrocyte precursor transplants are the best approach.

NeuralStem is developing human spinal cord-derived neural stem cells for ALS. The company expects to submit an IND submission in a few weeks and to begin clinical trials in early 2009.

"Our spinal cord-derived stem cells differentiate into about $75 \%$ spinal cord neurons and about $25 \%$ glia-most of which are astrocytes," he said. Thus, he said, the company's therapy already encompasses neuroprotection and restoration of function provided by astrocyte precursor transplants, as well as what is enabled by transplantation of neurons.

He also noted that about one-third of the cells transplanted in the Nature Neuroscience article survived. "We see the equivalent of nearly $100 \%$ survival of cells in our neural stem cell transplants," Garr said.

The number of cells that survive after transplantation has a direct impact on both the efficacy and safety of surgical implants, he said. For example, Garr said that 20 injections bilaterally of neural stem cells into the lower lumbar should be the maximum safe dose for preserving ambulation in patients, based on the company's safety studies in pigs. If a significant proportion of those cells did not survive, patients would have to undergo more than 20 injections, he said.

"So we think there is a clear correlation between cell survival and the number of injections a patient will need, and a clear correlation between the number of injections and possible benefit," Garr said.

Maragakis agreed with Garr that the astrocyte precursor therapy appeared to be dose limited.

In addition to the experiments in which 6 injections of 150,000 cells each showed good effects in rats, "we tried a kind of dose-response study by injecting about three times as many cells per site, but we observed necroses," as described in the Nature Neuroscience paper, he said.

The team thinks this resulted from putting too many cells in the small space of the spinal column, where "they form a ball with a center that has no nutrients or blood supply-so it becomes necrotic, much as a tumor center does," said Maragakis.

Nevertheless, Maragakis said astrocyte precursor therapy might be safer than other cell-based therapies. "The precursor cells are very restricted in terms of what kind of cells they can become," he noted. They cannot become neurons or other non-neural, nonbrain tissue-such as tumor cells - in the way a neural stem cell might," he said.

"Since our cellular therapeutic has the potential to become either astrocytes or oligodendrocytes, it could be effective both in neurodegenerative indications like ALS and in diseases of demyelination, such as transverse myelitis," said James Campanelli, Q Therapeutics' senior director of R\&D.

\section{Q-cell continuum}

In addition to working with Maragakis' group on ALS, Q Therapeutics is collaborating with Douglas Kerr at Johns Hopkins to develop Q-cells to treat transverse myelitis, a disease caused by an inflammatory, autoimmune attack at a discrete spot on the spinal column that demyelinates neurons and leaves the patient with a permanent disability.

Kerr is associate professor of neurology, molecular microbiology and immunology at Johns Hopkins and director of the Johns Hopkins Transverse Myelitis Center.

"These two diseases-ALS and transverse myelitis-are stepping stones towards applying this therapy to other diseases like multiple sclerosis, cerebral palsy, white matter stroke-and later, traumatic spinal cord injury," said Eppstein.

She also suggested the therapy might have utility in other neurodegenerative diseases that do not affect the spinal column, such as Parkinson's disease (PD).

Indeed, Maragakis' research group at Johns Hopkins is exploring other indications for which astrocyte precursor transplants might be effective.

He said that astrocytes are responsible for the uptake of excess glutamate, suggesting that "astrocyte precursors could be used to treat indications involving glutamate excitotoxicity, such as epilepsy and white matter stroke. We have looked at some models for this."

The team is also studying the role of glutamate and astrocytes in spinal cord injury (SCI).

Meanwhile, Rothstein's group at Johns Hopkins has been examining the role of GLT1 in ALS. "We think we've figured out the mechanism of GLT1 loss in ALS," which will be reported in an upcoming publication, he said.

Haas, M.J. SciBX 1(40); doi:10.1038/scibx.2008.962

Published online Nov. 6, 2008

\section{REFERENCES}

1. Lepore, A. et al. Nat. Neurosci.; published online Oct. 19, 2008; doi:10.1038/nn.2210

Contact: Nicholas Maragakis, Johns Hopkins University School of Medicine, Baltimore, Md.

e-mail: nmaragak@jhmi.edu

2. Yamanaka, K. et al. Nat. Neurosci. 11, 251-253 (2008)

3. Rao, M. et al. Proc. Natl. Acad. Sci. USA 95, 3996-4001 (1998)

\section{COMPANIES AND INSTITUTIONS MENTIONED}

Invitrogen Corp. (NASDAQ:IVGN), Carlsbad, Calif.

Johns Hopkins University, Baltimore, Md.

NeuralStem Inc. (AMEX:CUR), Rockville, Md.

Q Therapeutics Inc., Salt Lake City, Utah

sanofi-aventis Group (Euronext:SAN; NYSE:SNY), Paris, France

University of California, San Diego, Calif.

University of Utah School of Medicine, Salt Lake City, Utah 